\title{
Comparison of the Effects of Glutamine, Curcumin, and Nesfatin-1 on the Gastric Serosal Surface Neomucosa Formation: An Experimental Rodent Model
}

\author{
Osman Bilgin Gulcicek, ${ }^{1}$ Ali Solmaz, ${ }^{1}$ Hakan Yiğitbaş, ${ }^{1}$ Candas Ercetin, ${ }^{1}$ \\ Erkan Yavuz, ${ }^{1}$ Kamil Ozdogan, ${ }^{1}$ Sinan Arici, ${ }^{1}$ Asli Kahraman Akkalp, ${ }^{2}$ Tulin Sarac, ${ }^{3}$ \\ Fatih Celebi, ${ }^{1}$ and Atilla Celik ${ }^{1}$ \\ ${ }^{1}$ Department of General Surgery, Istanbul Bagcilar Training and Research Hospital, 34100 Istanbul, Turkey \\ ${ }^{2}$ Department of Pathology, Istanbul Bagcilar Training and Research Hospital, 34100 Istanbul, Turkey \\ ${ }^{3}$ Department of Biochemistry, Istanbul Bagcilar Training and Research Hospital, 34100 Istanbul, Turkey
}

Correspondence should be addressed to Osman Bilgin Gulcicek; drosmanbilgin@hotmail.com

Received 3 May 2016; Accepted 28 June 2016

Academic Editor: Paolo Gionchetti

Copyright ( $) 2016$ Osman Bilgin Gulcicek et al. This is an open access article distributed under the Creative Commons Attribution License, which permits unrestricted use, distribution, and reproduction in any medium, provided the original work is properly cited.

Introduction. Short bowel syndrome can crop up if more than $50 \%$ of small intestine is resected or when less than $100 \mathrm{~cm}$ of small bowel is left. Glutamine is the main food source of enterocytes. Curcumin has protective effects on intestinal ischemia-reperfusion damage. Nesfatin-1 is a satiety molecule. It has protective effects on gastric mucosa. The primary purpose of this study is to compare effects of glutamine, curcumin, and nesfatin-1 on the gastric serosal surface neomucosa formation on rats. Materials and Methods. 24 Wistar-Hannover rats were randomly divided into 4 groups and treated with saline, glutamine, curcumin, and nesfatin-1 after ileogastric anastomosis. After 14 days all rats were euthanized, and blood was collected. En bloc resection of anastomotic part was performed for histopathological examination. Results. PDGF, TGF- $\beta$, and VEGF levels and neomucosa formation were higher in glutamine group $(p=0.003, p=0.003$, and $p=0.025)$. Glutamine promotes the intestinal neomucosa formation on the gastric serosal surface and augments growth factors essential for neomucosa formation on rats. Conclusion. Glutamine may be used in short bowel syndrome for increasing the absorption surface area. But that needs to be determined by adequately powered clinical trials.

\section{Introduction}

Short bowel syndrome (SBS) is the clinical sequela of inefficient absorption of nutrient and fluid from small intestine that can be congenital or acquired and it is characterized with reduced small bowel length [1]. It is the main cause of intestinal failure (IF) seen after intestinal resection [2] and IF is a term generally used with the SBS [3]. The syndrome is characterized by maldigestion, malabsorption, and malnutrition [4]. Incidence and prevalence are estimated as 3 per million and 4 per million, respectively [5].

The most common causes of SBS are small bowel atresia, aganglionosis, gastroschisis, necrotizing enterocolitis, volvulus, and intussusception in children [6]. Strangulated bowel,
Crohn's disease, ischemia, and trauma are the most common factors leading to SBS in adults [4]. SBS is the most common cause of pediatric IF and is associated with significant morbidity and mortality [7]. These patients present symptoms including diarrhea, steatorrhea, abdominal pain, malnutrition, and dehydration. The severity of symptoms depends on the length of small bowel remaining [8]. SBS may crop up after the resection of more than $50 \%$ of small intestine and certainly emerges after resection of more than $70 \%$ or when less than $100 \mathrm{~cm}$ of small bowel is left [9].

The duodenum and jejunum are the primary sites of protein, carbohydrate, fat, water soluble vitamin, and mineral absorption. Half of the jejunum can be removed without significant problem [10]. However resection of the ileum, 
particularly the terminal ileum, is more detrimental than loss of jejunum, because it is the only site for absorption of intrinsic factor bound B-12 and bile salts [11].

The remaining small bowel undergoes an adaptation process of three phases after resection. The acute phase is the stabilization which starts after resection and lasts less than 4 weeks. The second phase is the adaptation, which lasts 1-2 years. The last phase is the maintenance that requires permanent dietetic treatment $[12,13]$.

In the final phase of SBS, patients become dependent on long-term parenteral nutrition. However the optimization of intestinal digestion and absorption can be achieved by surgical treatment that consists of reconstructive procedures of remnant bowel and intestinal transplantation [14]. The aim of reconstructive procedures is slowing down the intestinal transit time and the development of new intestinal mucosa, neomucosa. All procedures are still experimental [15].

Glutamine is the primary metabolic fuel of rapidly dividing cells such as small intestinal enterocytes. It also stimulates proliferation of these cells $[16,17]$. Previous studies have shown that glutamine has antioxidant effects and immune modulation properties [18]. Curcumin is antioxidant and anti-inflammatory agent whose protective effects on intestinal ischemia-reperfusion damage have been shown in the recent studies [19]. Nesfatin-1 is a recently identified satietyinducing molecule derived from nucleobindin-2 (NUCB2) in hypothalamic nuclei. It also has anti-inflammatory, antiapoptotic, and protective effects on gastric mucosa [20, 21]. Recently published studies have demonstrated its antiinflammatory effects via the maintenance of the intracellular antioxidants [22].

The use of serosal patching is a technique to grow new intestinal mucosa. Growing neomucosa is used for enlargement of absorptive surface, but it is still experimental [15].

In this experimental study, our aim was to investigate the potential effects of glutamine, curcumin, and nesfatin1 on the neomucosa formation of the serosal surface of the stomach that was used as a patch to terminal ileal defect on rats.

\section{Materials and Methods}

2.1. Experimental Design. Twenty-four male Wistar-Hannover rats (300-500 g), obtained from Bagcilar Training and Research Hospital Animal Center (BADABEM), were housed in cages under controlled room temperature $\left(21 \pm 2^{\circ} \mathrm{C}\right)$ and humidity (60-70\%) with $12 \mathrm{~h}$ light-dark schedule and were fed with standard pellet, ad libitum (MBD Animal Feed, Kocaeli, Turkey). All experimental procedures were approved by the Bagcilar Training and Research Hospital Animal Care and Use Committee (2014-01).

2.2. Chemicals and Reagents. The curcuminoid mixture, purchased from Sigma (Sigma, C1386, St. Louis, MO, USA), was identified as curcumin. The authentic curcuminoids were dissolved in corn oil at a concentration of $1 \mathrm{mg} / \mathrm{mL}$ in brown glass vials and stored at $4^{\circ} \mathrm{C}$. Reagents were obtained from Merck (Darmstadt, Germany). Nesfatin-1 (Bioss, Beijing,
China) was dissolved in distilled water and injected intraperitoneally. Glutamine was purchased from Nestle (Resource Glutamine Şase 5 gr; Nestle Healthcare Nutrition, Germany).

2.3. Study Groups and Treatment. Rats were randomly divided into 4 groups ( 8 in each). Group 1 (control) was treated with saline after ileogastric anastomosis between mucosal surface of the ileum and serosal surface of the stomach. Group 2 was treated with glutamine $(4 \mathrm{~mL} / \mathrm{kg} / \mathrm{day}$, by gavage) after the same anastomosis. Group 3 was treated with curcumin ( $2 \mathrm{~mL} / \mathrm{kg} /$ day, by gavage) after the same anastomosis. Group 4 was treated with nesfatin-1 $(2 \mu \mathrm{g} / \mathrm{kg} / \mathrm{day}$, intraperitoneally). After 14 days all rats were euthanized under anesthesia, and blood was collected by cardiac puncture, centrifuged, and stored at $-80^{\circ} \mathrm{C}$ for the measurement of vascular endothelial growth factor (VEGF), platelet derived growth factor (PDGF), fibroblast growth factor (FGF), transforming growth factor beta (TGF- $\beta$ ), and epidermal growth factor (EGF).

After midline laparotomy en bloc resection of anastomotic part of terminal ileum and stomach was performed and washed with saline and tissues were fixed in $10 \%$ formaldehyde solution for histopathological examination (Figure 1).

2.4. Surgical Procedure. Rats were anesthetized by an isoflurane (5\% for induction and $2 \%$ for maintenance, İsoflurane ${ }^{\circledR}$; Baxter, Puerto Rico, USA). Under aseptic conditions $3 \mathrm{~cm}$ midline abdominal incision was done. A one $\mathrm{cm}$ longitudinal incision was done in the terminal ileal region and the anastomosis between mucosal surface of the terminal ileum and the serosal surface of the stomach was performed with continuous 6.0 polypropylene sutures (Dogsan, Trabzon, Turkey).

2.5. Histological Analysis. The anastomotic parts were fixed in $10 \%$ formaldehyde and routinely processed for paraffin embedding. Four-micron-thick paraffin sections were obtained and stained with hematoxylin-eosin for the evaluation of inflammatory process, granulation tissue and neomucosa formation, and scoring (0: none, 1: mild, 2: moderate, and 3: severe). Masson's trichrome staining was performed for examination of fibroblastic activity of the neomucosal region, Alcian Blue ( $\mathrm{pH}$ : 2.5) staining for the examination of intestinal type mucin (Figure 2).

2.6. Biochemical Analyses. Blood samples were centrifuged for 10 minutes in $4000 \mathrm{rpm}$ at $+4^{\circ} \mathrm{C}$. Serum samples were separated into portions and stored at $-80^{\circ} \mathrm{C}$. Plasma levels of PDGF, VEGF, TGF- $\beta$, NO, EGF, and FGF were quantified by using enzyme-linked immunosorbent assay (ELISA) kits specific for the rat, according to the manufacturers' instructions and guidelines (Sunredbio, Shanghai, China). These particular assay kits were chosen because of their high degree of sensitivity and selectivity and inter- and intraassay precision and the small amount of plasma sample required to conduct the assay. Elisa assays were performed with Biotek GEN5 calculation program by using Biotek ELx800 microplate reader and Biotek ELx-50 microplate washer. 


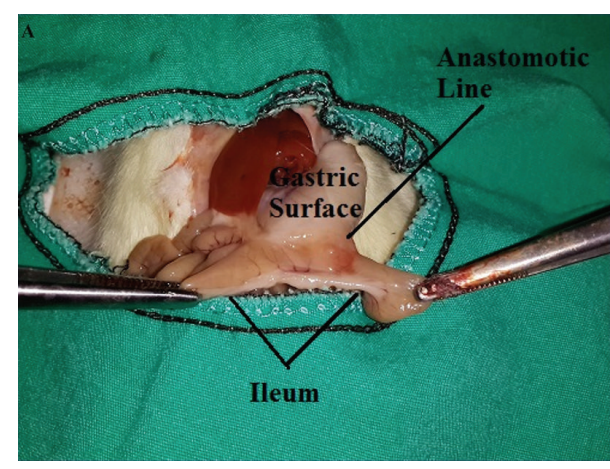

(a)

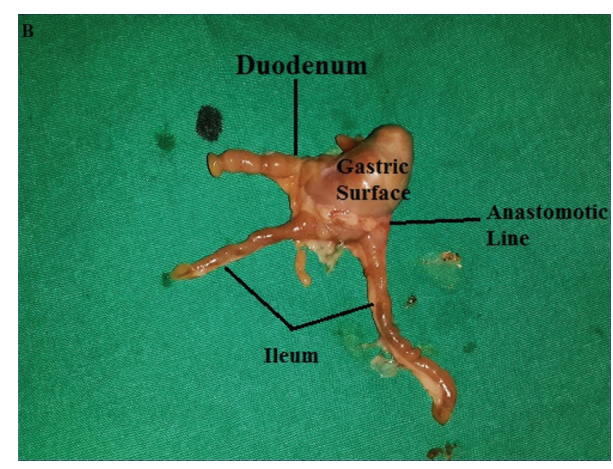

(b)

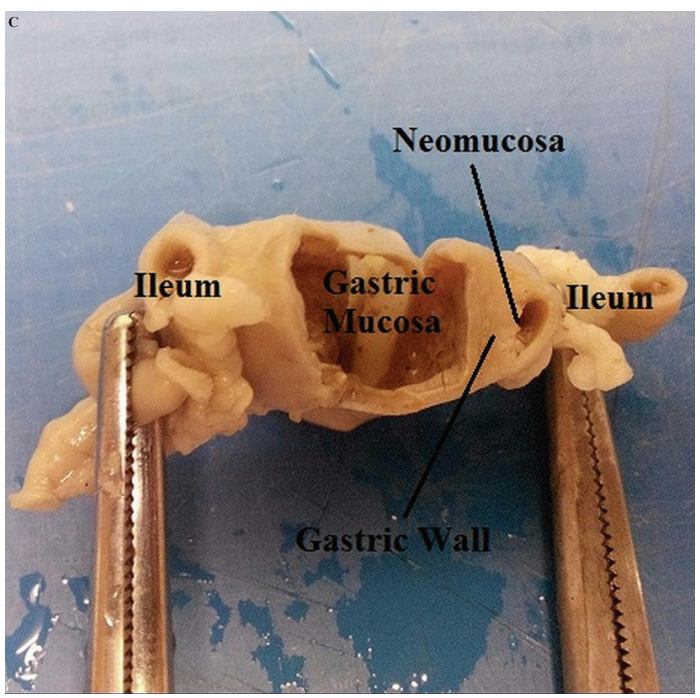

(c)

FIGURE 1: Surgical procedure and histopathological assessment. ((a) and (b)) Anastomotic line is shown between the gastric surface and ileum on the postoperative 14th day. (c) Neomucosa formation on the gastric surface area.

2.7. Statistical Analyses. Statistical analysis was performed by using the NCSS (Number Cruncher Statistical System) 2007 Statistical Software (Utah, USA) package program. Descriptive statistical methods (mean, standard deviation), Kruskal-Wallis test (for the group comparison), Dunn's multiple comparison test (for subgroup analysis), and Chi-square test (for comparison of qualitative data) were used. A $p$ value of $<0.05$ was considered statistically significant.

\section{Results}

3.1. Biochemical Evaluation. There were no significant differences between nesfatin-1, curcumin, glutamine, and control groups in terms of EGF and FGF levels $(p=0.082, p=$ 0.076 ) (Table 1). Contrarily, there was significant difference between groups in terms of PDGF, TGF- $\beta$, and VEGF $(p<$ $0.05)$. PDGF level was significantly higher in glutaminetreated group than others $(p=0.013, p=0.025$, and $p=0.004)$. Glutamine- and curcumin-treated groups had significantly higher TGF- $\beta$ levels compared to control group ( $p=0.037, p=0.01)$; however TGF- $\beta$ levels of nesfatin1-treated group were similar to control group $(p=0.337)$.
There was no significant difference between glutamine- and curcumin-treated groups in terms of TGF- $\beta$ levels ( $p=$ 0.749). VEGF levels of glutamine- and curcumin-treated groups were significantly higher than control group $(p=$ 0.016, $p=0.025)$; however nesfatin-1-treated group had nonsignificant results compared to control group $(p=0.749)$. Glutamine-treated group and curcumin-treated group had similar VEGF levels $(p=0.998)$ (Table 2$)$.

3.2. Histological Evaluation. There was no significant difference between all groups in terms of inflammatory process, granulation tissue formation, fibroblastic activity, and neomucosa formation histologically $(p>0.05)$ (Table 3$)$. Neomucosa formation was determined in 2 rats of control group (33.33\%), 5 rats of glutamine-treated group (83.33\%), 3 rats of curcumin-treated group (50\%), and 4 rats of nesfatin1-treated group (66.67\%) (Figure 3).

\section{Discussion}

Intestinal failure has been defined as the inability of gastrointestinal tract to sustain adequate digestion and absorption 

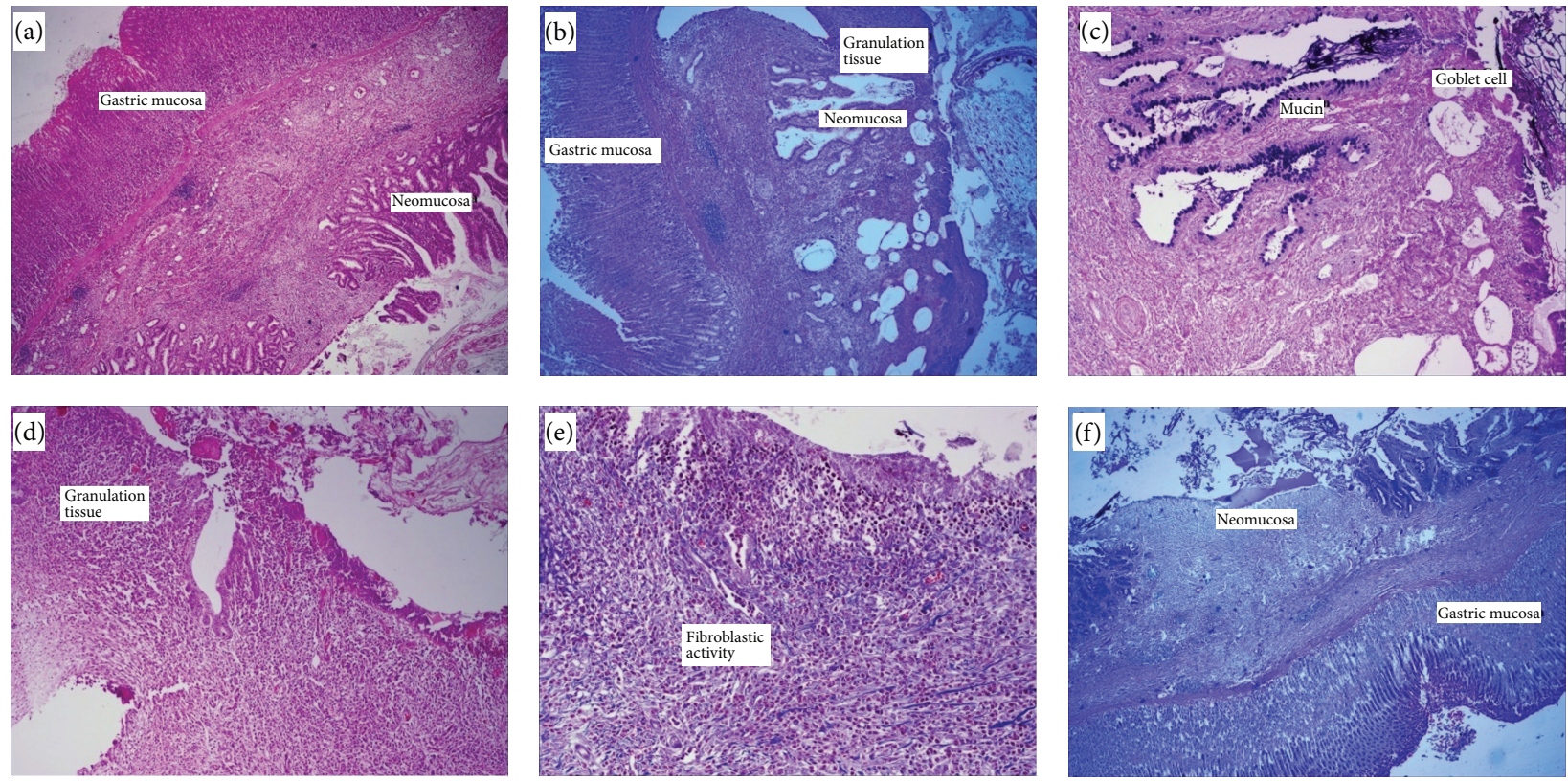

Figure 2: Histological and morphologic evaluation. ((a) and (b)) Gastric mucosa, granulation tissue, and neomucosal tissue formation [hematoxylin and eosin $(\mathrm{HE}) \times 110$ ]. (c) Goblet cells and mucin in neomucosal surface [Alcian Blue $(\mathrm{AB}) \times 220$ ]. (d) Ulcer and granulation tissue in neomucosal surface $[\mathrm{HE} \times 110]$. (e) Fibroblastic activity in anastomotic line [Mason Trichrome $\times 110]$. (f) Newly formed neomucosa and gastric mucosa $[$ Alcian Blue $\times 110]$.

TABLE 1: Average EGF, FGF, PDGF, TGF- $\beta$, and VEGF levels (pg/mL).

\begin{tabular}{|c|c|c|c|c|c|}
\hline & Control group & Glutamine group & Curcumin group & Nesfatin-1 group & $p$ \\
\hline EGF & $246.39 \pm 40.1$ & $293.55 \pm 54.09$ & $306.16 \pm 45.54$ & $227.08 \pm 61.35$ & 0.082 \\
\hline FGF & $82.57 \pm 8.73$ & $89.07 \pm 15.37$ & $94.97 \pm 6.11$ & $77.03 \pm 14.02$ & 0.076 \\
\hline PDGF & $2.38 \pm 0.21$ & $3.33 \pm 0.52$ & $2.66 \pm 0.42$ & $2.1 \pm 0.31$ & 0.003 \\
\hline TGF- $\beta$ & $725.74 \pm 89.07$ & $859.21 \pm 93.05$ & $877.35 \pm 66.08$ & $631.72 \pm 166.13$ & 0.003 \\
\hline VEGF & $264.16 \pm 43.5$ & $338.49 \pm 50.11$ & $346.6 \pm 57.73$ & $261.27 \pm 71.75$ & 0.025 \\
\hline
\end{tabular}

without parenteral nutrition. Short bowel syndrome is the most common cause of intestinal failure in children [23]. The management of this syndrome requires a multidisciplinary approach with parenteral nutrition and sometimes surgery [24]. These complex treatment modalities are associated with significant morbidity and mortality rates [25].

SBS is the primary reason for patients to receive longterm parenteral nutrition (PN). PN brings many complications such as life-threatening infections, catheter malfunction, venous thromboembolism, and metabolic complications like liver and renal disease and eventually organ failure $[26,27]$.

There are several surgical options for the management of SBS, including construction of intestinal valves or reversed intestinal segments, colon interposition, and lengthening procedures [24]. However success rates of these surgical procedures are still limited.

The current surgical approaches for SBS are intestinal transplantation and autologous reconstruction procedures consisting of growing mucosal surface area and lengthening bowel [24]. The main autologous intestinal reconstruction procedures are the Longitudinal Intestinal Lengthening and
Tailoring (LILT) known as Bianchi Procedure and the Serial Transverse Enteroplasty (STEP) [28]. Nontransplant surgical approaches (LILT and STEP procedures) are both accepted modalities for the elimination of parenteral nutrition dependence [29]. The LILT procedure was firstly described by Bianchi in 1980. Both procedures have serious complications like stricture, leakage, and bleeding [29].

Intestinal transplantation is the last option for SBS. It has some serious complications and should be performed at specialized centers by experienced surgeons. Morbidity and mortality rates of intestinal transplantation are still very high all over the world [29].

Growing neomucosa is a technique, aiming for augmentation of absorptive surface of the intestinal mucosa; however it is still performed experimentally [15]. The use of serosal patching to grow new intestinal mucosa is a technique used for enlargement of the mucosal surface. The regenerated intestinal mucosa develops by lateral ingrowth of the adjacent mucosa and has same functions as normal intestinal mucosa [30].

There are same experimental models for the growing of the neomucosa by using serosal patch technique in the 
TABLE 2: PDGF, TGF- $\beta$, and VEGF levels.

\begin{tabular}{lccc}
\hline & PDGF $(p$ value $)$ & TGF- $\beta(p$ value $)$ & VEGF $(p$ value $)$ \\
\hline Control group/nesfatin-1 group & 0.055 & 0.337 & 0.749 \\
Control group/glutamine group & $\mathbf{0 . 0 1 3}$ & $\mathbf{0 . 0 3 7}$ & $\mathbf{0 . 0 1 6}$ \\
Control group/curcumin group & 0.262 & $\mathbf{0 . 0 1}$ & $\mathbf{0 . 0 2 5}$ \\
Nesfatin-1 group/glutamine group & $\mathbf{0 . 0 0 4}$ & $\mathbf{0 . 0 1}$ & 0.109 \\
Nesfatin-1 group/curcumin group & $\mathbf{0 . 0 3 7}$ & $\mathbf{0 . 0 0 4}$ & $\mathbf{0 . 0 2 5}$ \\
Glutamine group/curcumin group & $\mathbf{0 . 0 2 5}$ & 0.749 & 0.998 \\
\hline
\end{tabular}

TABLE 3: Percentage of inflammatory process, granulation of tissue formation, fibroblastic activity, and neomucosa formation.

\begin{tabular}{|c|c|c|c|c|c|c|c|c|c|c|}
\hline & & \multicolumn{2}{|c|}{ Control group } & \multicolumn{2}{|c|}{ Glutamine group } & \multicolumn{2}{|c|}{ Curcumin group } & \multicolumn{2}{|c|}{ Nesfatin-1 group } & \multirow[t]{2}{*}{$p$} \\
\hline \multirow{4}{*}{ Inflammatory process } & Minimal & 2 & $33,33 \%$ & 2 & $33,33 \%$ & 2 & $33,33 \%$ & 0 & $0,00 \%$ & \\
\hline & Mild & 2 & $33,33 \%$ & 2 & $33,33 \%$ & 0 & $0,00 \%$ & 1 & $16,67 \%$ & \multirow{3}{*}{0.481} \\
\hline & Moderate & 2 & $33,33 \%$ & 2 & $33,33 \%$ & 4 & $66,67 \%$ & 4 & $66,67 \%$ & \\
\hline & Severe & 0 & $0,00 \%$ & 0 & $0,00 \%$ & 0 & $0,00 \%$ & 1 & $16,67 \%$ & \\
\hline \multirow{3}{*}{ Granulation tissue form } & Minimal & 1 & $16,67 \%$ & 2 & $2,00 \%$ & 2 & $33,33 \%$ & 0 & $0,00 \%$ & \multirow{3}{*}{0.401} \\
\hline & Mild & 4 & $66,67 \%$ & 3 & $50,00 \%$ & 2 & $33,33 \%$ & 2 & $33,33 \%$ & \\
\hline & Moderate & 1 & $16,67 \%$ & 1 & $16,67 \%$ & 2 & $33,33 \%$ & 4 & $66,67 \%$ & \\
\hline \multirow{4}{*}{ Fibroblastic activity } & Minimal & 1 & $16,67 \%$ & 2 & $33,33 \%$ & 1 & $16,67 \%$ & 0 & $0,00 \%$ & \multirow{4}{*}{0.242} \\
\hline & Mild & 1 & $16,67 \%$ & 3 & $50,00 \%$ & 5 & $83,33 \%$ & 3 & $50,00 \%$ & \\
\hline & Moderate & 3 & $50,00 \%$ & 1 & $16,67 \%$ & 0 & $0,00 \%$ & 3 & $50,00 \%$ & \\
\hline & Severe & 1 & $16,67 \%$ & 0 & $0,00 \%$ & 0 & $0,00 \%$ & 0 & $0,00 \%$ & \\
\hline \multirow{2}{*}{ Neo. } & Absent & 4 & $66,67 \%$ & 1 & $16,67 \%$ & 3 & $50,00 \%$ & 2 & $33,33 \%$ & \multirow{2}{*}{0.33} \\
\hline & Present & 2 & $33,33 \%$ & 5 & $83,33 \%$ & 3 & $50,00 \%$ & 4 & $66,67 \%$ & \\
\hline
\end{tabular}

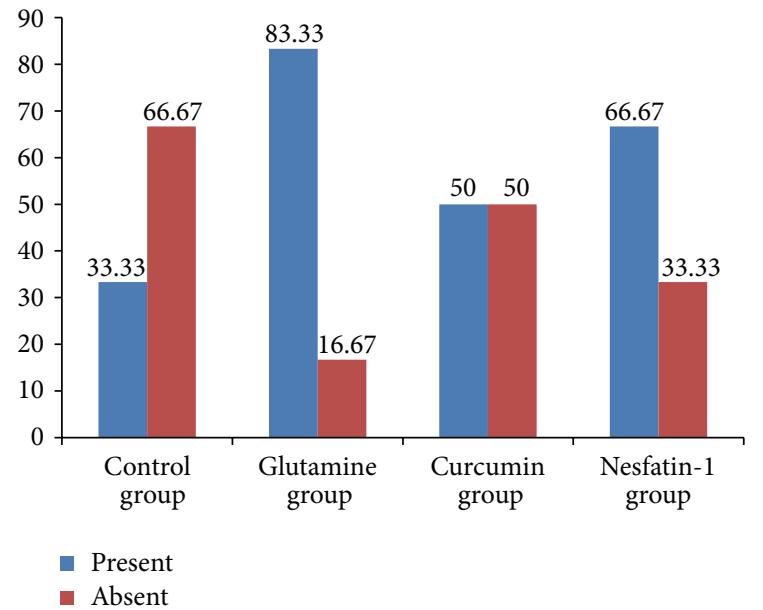

FIGURE 3: Ratio of neomucosa formation in groups (\%).

literature [31]. Serosal surface of the small bowel, colon, and peritoneal surface have been used as a serosal patch [32]. Saday and Mir used intestinal surface as a patch in a rabbit model and the neomucosa grown on the serosal side of the common wall of intestine [33].

According to the review published by Freud and Eshet, urogastrone, octreotide, epidermal growth factor (EGF), and prostaglandin E2 analogues have beneficial effects on the neomucosa formation on the serosal patch [31].
Recently it has been suggested by some authors that transforming growth factor beta (TGF- $\beta$ ), growth hormone, and epidermal growth factor (EGF) have favorable effects on bowel mucosa [34]. Glutamine is the primary fuel source for rapidly dividing cells like enterocytes and it prevents intestinal atrophy [35]. Adas et al. showed efficacious effects of hyperbaric oxygen and growth hormone together on the neomucosa formation in the gastric surface patch model [36].

Epidermal growth factor (EGF) is a 53-amino-acid polypeptide which has a mitogenic and cytoprotective role. It stimulates the formation of granulation tissue, epithelial cell migration and proliferation, and formation of angiogenesis [37]. Fibroblast growth factor (FGF) regulates the tissue homeostasis and vascular branching morphogenesis and also increases the levels of transforming growth factor beta (TGFв) [38].

In our study EGF and FGF values of glutamine- and curcumin-treated groups were higher than nesfatin-1 and control groups (Table 1).

Platelet derived growth factor (PDGF) regulates the cellular proliferation and angiogenesis and stimulates the formation of connective tissue matrix, collagen, glycosaminoglycans, and proteoglycans. PDGF promotes the remodeling of soft tissue [39]. Transforming growth factor beta (TGF$\beta$ ) plays an important role in cellular proliferation process, tissue repair, and inflammatory responses. TGF- $\beta$ promotes the expression of type 1 collagen, type 5 collagen, and proteoglycans [40]. Vascular endothelial growth factor (VEGF) stimulates the endothelial cell proliferation and induces 
angiogenesis. It has an important role in neovascularization [41].

In present study glutamine-treated group had significantly higher levels of PDGF, TGF- $\beta$, and VEGF. But glutamine and curcumin groups were similar in terms of TGF- $\beta$ and VEGF levels $(p=0.74, p=0.99)$.

Although histopathological examination revealed that there was no significant difference between the groups in terms of inflammatory process, formation of granulation tissue, fibroblastic activity, and neomucosa formation in this study, glutamine-treated group had highest percentage $(83.3 \%, n=5)$ of neomucosa formation.

Present study is also a surface expander study. And it is supported that intestinal neomucosa can be successfully raised on gastric serosal surface. So regenerative and absorptive capability of intestinal mucosa increase. Also the role of glutamine on intestinal neomucosa formation on gastric serosa was investigated in short bowel syndrome.

In conclusion, glutamine promotes the intestinal neomucosa formation on gastric serosal surface and augments growth factors which were essential for neomucosa formation in rats. Glutamine may be used in short bowel syndrome for increasing the absorption surface area. But that needs to be determined by adequately powered clinical trials.

This study was limited by the small number of rats and short period of the experiment. More meaningful results can be obtained by using a greater number of rats and extending the duration of experiment period. So this study encourages us for further studies.

\section{Disclosure}

A part of this study, with preliminary data, was presented in 7th European Multidisciplinary Colorectal Cancer Congress, Amsterdam, 2014 (poster presentation).

\section{Competing Interests}

The authors declare that they have no competing interests.

\section{Acknowledgments}

The present work was supported by the Istanbul Bagcilar Training and Research Hospital Educational Planning and Coordination Committee (Project no. 2014-01).

\section{References}

[1] S. M. Wilhelm, M. Lipari, J. K. Kulik, and P. B. Kale-Pradhan, "Teduglutide for the treatment of short bowel syndrome," Annals of Pharmacotherapy, vol. 48, no. 9, pp. 1209-1213, 2014.

[2] P. B. Jeppesen, "New approaches to the treatments of short bowel syndrome-associated intestinal failure," Current Opinion in Gastroenterology, vol. 30, no. 2, pp. 182-188, 2014.

[3] A. L. Buchman, J. Scolapio, and J. Fryer, "AGA technical review on short bowel syndrome and intestinal transplantation," Gastroenterology, vol. 124, no. 4, pp. 1111-1134, 2003.

[4] C. E. Semrad, "Approach to the patient with diarrhea and malabsorption," in Goldman's Cecil Medicine, pp. 895-913, Elsevier Saunders, Philadelphia, Pa, USA, 24th edition, 2012.
[5] B. Z. Godoy, J. Faintuch, M. L. Marin, M. A. Nogueira, V. B. Pinto, and W. M. Pollara, "Off label pharmacological therapy in patients with short bowel syndrome," European Review for Medical and Pharmacological Sciences, vol. 17, no. 24, pp. 32853290, 2013.

[6] O. Goulet and F. Ruemmele, "Causes and management of intestinal failure in children," Gastroenterology, vol. 130, no. 2, pp. S16-S28, 2006.

[7] T. Wester, H. Borg, H. Naji, P. Stenström, G. Westbacke, and H. E. Lilja, "Serial transverse enteroplasty to facilitate enteral autonomy in selected children with short bowel syndrome," British Journal of Surgery, vol. 101, no. 10, pp. 1329-1333, 2014.

[8] D. G. Kelly, K. A. Tappenden, and M. F. Winkler, "Short bowel syndrome: highlights of patient management, quality of life, and survival," Journal of Parenteral and Enteral Nutrition, vol. 38, no. 4, pp. 427-437, 2014.

[9] B. D. Yildiz, "Where are we at with short bowel syndrome and small bowel transplant," World Journal of Transplantation, vol. 2, no. 6, pp. 95-103, 2012.

[10] K. N. Jeejeebhoy, "Short bowel syndrome: a nutritional and medical approach," Canadian Medical Association Journal, vol. 166, no. 10, pp. 1297-1302, 2002.

[11] E. A. Wall, "An overview of short bowel syndrome management: adherence, adaptation, and practical recommendations," Journal of the Academy of Nutrition and Dietetics, vol. 113, no. 9, pp. 1200-1208, 2013.

[12] J. Keller, H. Panter, and P. Layer, "Management of the short bowel syndrome after extensive small bowel resection," Best Practice and Research: Clinical Gastroenterology, vol. 18, no. 5, pp. 977-992, 2004.

[13] J. E. Bines, "Intestinal failure: a new era in clinical management," Journal of Gastroenterology and Hepatology, vol. 24, supplement 3, pp. S86-S92, 2009.

[14] P. B. Jeppesen, "Modern treatment of short bowel syndrome," Current Opinion in Clinical Nutrition and Metabolic Care, vol. 16 , no. 5, pp. 582-587, 2013.

[15] A. J. W. Millar, "Non-transplant surgery for short bowel syndrome," Pediatric Surgery International, vol. 29, no. 10, pp. 983987, 2013.

[16] A.-L. Wang, Q. Niu, N. Shi et al., "Glutamine ameliorates intestinal ischemia-reperfusion injury in rats by activating the Nrf2/Are signaling pathway," International Journal of Clinical and Experimental Pathology, vol. 8, no. 7, pp. 7896-7904, 2015.

[17] D. Yao, L. Zheng, J. Wang, M. Guo, J. Yin, and Y. Li, "Perioperative alanyl-glutamine-supplemented parenteral nutrition in chronic radiation enteritis patients with surgical intestinal obstruction: a prospective, randomized, controlled study," Nutrition in Clinical Practice, vol. 31, no. 2, pp. 250-256, 2016.

[18] C.-C. Lai, W.-L. Liu, and C.-M. Chen, "Glutamine attenuates acute lung injury caused by acid aspiration," Nutrients, vol. 6, no. 8, pp. 3101-3116, 2014.

[19] O. Karatepe, O. B. Gulcicek, M. Ugurlucan et al., "Curcumin nutrition for the prevention of mesenteric ischemia-reperfusion injury: an experimental rodent model," Transplantation Proceedings, vol. 41, no. 9, pp. 3611-3616, 2009.

[20] A. Szlachcic, Z. Sliwowski, G. Krzysiek-Maczka et al., "New satiety hormone nesfatin-1 protects gastric mucosa against stress-induced injury: mechanistic roles of prostaglandins, nitric oxide, sensory nerves and vanilloid receptors," Peptides, vol. 49, pp. 9-20, 2013. 
[21] D. Özsavcí, M. Erşahin, A. Şener et al., "The novel function of nesfatin-1 as an anti-inflammatory and antiapoptotic peptide in subarachnoid hemorrhage-induced oxidative brain damage in rats," Neurosurgery, vol. 68, no. 6, pp. 1699-1708, 2011.

[22] M. Kolgazi, C. Cantali-Ozturk, R. Deniz et al., "Nesfatin-1 alleviates gastric damage via direct antioxidant mechanisms," Journal of Surgical Research, vol. 193, no. 1, pp. 111-118, 2015.

[23] I. M. Gutierrez, K. H. Kang, and T. Jaksic, "Neonatal short bowel syndrome," Seminars in Fetal and Neonatal Medicine, vol. 16, no. 3, pp. 157-163, 2011.

[24] J. Sommovilla and B. W. Warner, "Surgical options to enhance intestinal function in patients with short bowel syndrome," Current Opinion in Pediatrics, vol. 26, no. 3, pp. 350-355, 2014.

[25] B. P. Modi, M. Langer, Y. A. Ching et al., "Improved survival in a multidisciplinary short bowel syndrome program," Journal of Pediatric Surgery, vol. 43, no. 1, pp. 20-24, 2008.

[26] P. B. Jeppesen, M. Staun, and P. B. Mortensen, "Adult patients receiving home parenteral nutrition in Denmark from 1991 to 1996: who will benefit from intestinal transplantation?" Scandinavian Journal of Gastroenterology, vol. 33, no. 8, pp. 839846, 1998.

[27] M. Ferrone and J. S. Scolapio, "Teduglutide for the treatment of short bowel syndrome," Annals of Pharmacotherapy, vol. 40, no. 6, pp. 1105-1109, 2006.

[28] K. Reinshagen, K. Zahn, C. von Buch et al., "The impact of longitudinal intestinal lengthening and tailoring on liver function in short bowel syndrome," European Journal of Pediatric Surgery, vol. 18, no. 4, pp. 249-253, 2008.

[29] G. Frongia, M. Kessler, S. Weih, A. Nickkholgh, A. Mehrabi, and S. Holland-Cunz, "Comparison of LILT and STEP procedures in children with short bowel syndrome-a systematic review of the literature," Journal of Pediatric Surgery, vol. 48, no. 8, pp. 17941805, 2013.

[30] J. S. Thompson, "Neomucosal growth in serosa lined intestinal tunnels," Journal of Surgical Research, vol. 49, no. 1, pp. 1-7, 1990.

[31] E. Freud and R. Eshet, "Insights from animal models for growing intestinal neomucosa with serosal patching-a still untapped technique for the treatment of short bowel syndrome," Laboratory Animals, vol. 35, no. 2, pp. 180-187, 2001.

[32] F. C. Campbell, D. Smith, B. Waldron et al., "Mucosal function after ileal mucosal fenestration and colonic autotransplantation," British Journal of Surgery, vol. 78, no. 11, pp. 1309-1312, 1991.

[33] C. Saday and E. Mir, "A surgical model to increase the intestinal absorptive surface: intestinal lengthening and growing neomucosa in the same approach," Journal of Surgical Research, vol. 62, no. 2, pp. 184-191, 1996.

[34] M. Guclu, B. Demirogullari, S. Barun et al., "The effects of melatonin on intestinal adaptation in a rat model of short bowel syndrome," European Journal of Pediatric Surgery, vol. 24, no. 2, pp. 150-157, 2014.

[35] M. Guo, Y. Li, and J. Li, "Role of growth hormone, glutamine and enteral nutrition in pediatric short bowel syndrome: a pilot follow-up study," European Journal of Pediatric Surgery, vol. 22, no. 2, pp. 121-126, 2012.

[36] G. Adas, M. Adas, S. Arikan et al., "Effect of growth hormone, hyperbaric oxygen and combined therapy on the gastric serosa," World Journal of Gastroenterology, vol. 19, no. 19, pp. 2904-2912, 2013.

[37] B. M. Ertugrul, C. Buke, O. S. Ersoy, B. Ay, D. S. Demrez, and O. Savk, "Intralesional epidermal growth factor for diabetic foot wounds: the first cases in Turkey," Diabetic Foot and Ankle, vol. 6, Article ID 28419, 2015.

[38] L. Zheng, Q. Hui, L. Tang et al., "TAT-mediated acidic fibroblast growth factor delivery to the dermis improves wound healing of deep skin tissue in rat," PLoS ONE, vol. 10, no. 8, Article ID e0135291, 2015.

[39] E. Borkham-Kamphorst and R. Weiskirchen, "The PDGF system and its antagonists in liver fibrosis," Cytokine \& Growth Factor Reviews, vol. 28, pp. 53-61, 2016.

[40] B. Xie, C. Zhang, K. Kang, and S. Jiang, "miR-599 inhibits vascular smooth muscle cells proliferation and migration by targeting TGFB2," PLoS ONE, vol. 10, no. 11, Article ID e0141512, 2015.

[41] J. Zhang, X. Jiang, Y. Jiang et al., "Recent advances in the development of dual VEGFR and c-Met small molecule inhibitors as anticancer drugs," European Journal of Medicinal Chemistry, vol. 108, pp. 495-504, 2016. 


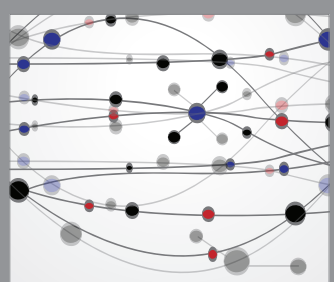

The Scientific World Journal
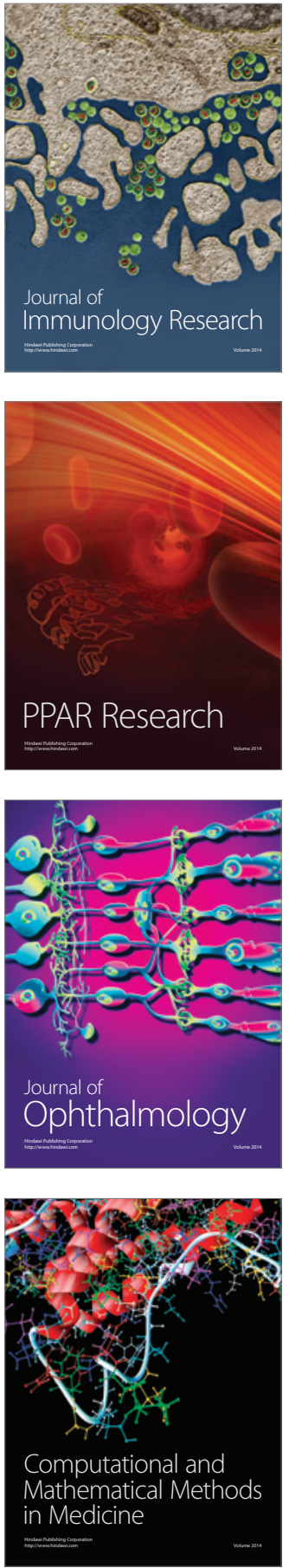

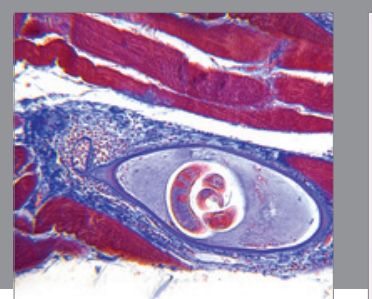

Gastroenterology Research and Practice

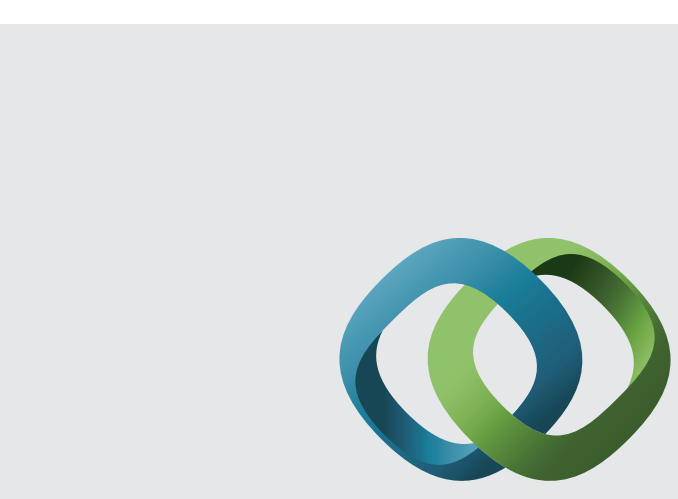

\section{Hindawi}

Submit your manuscripts at

http://www.hindawi.com
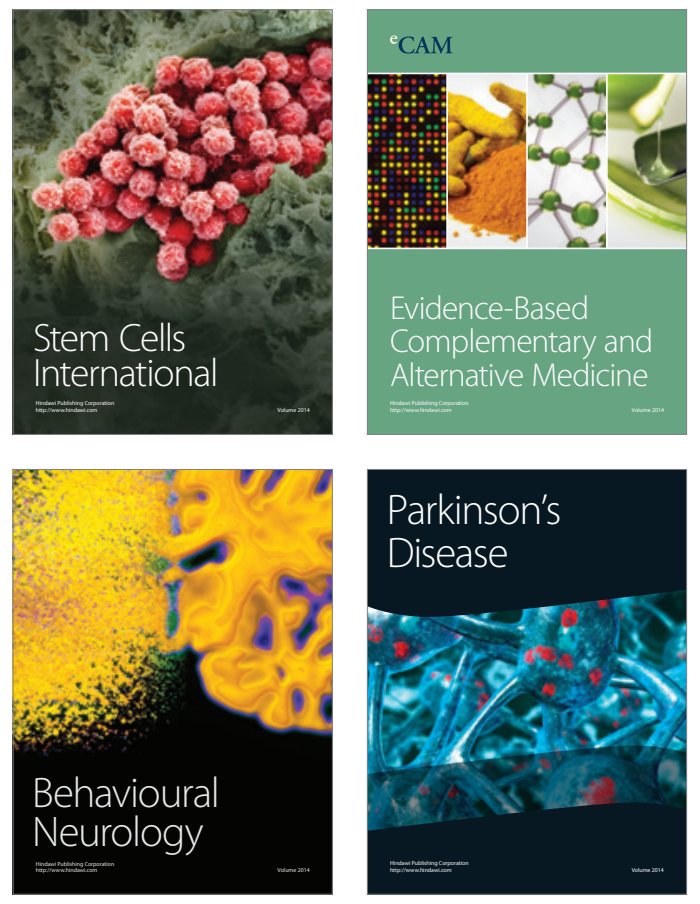
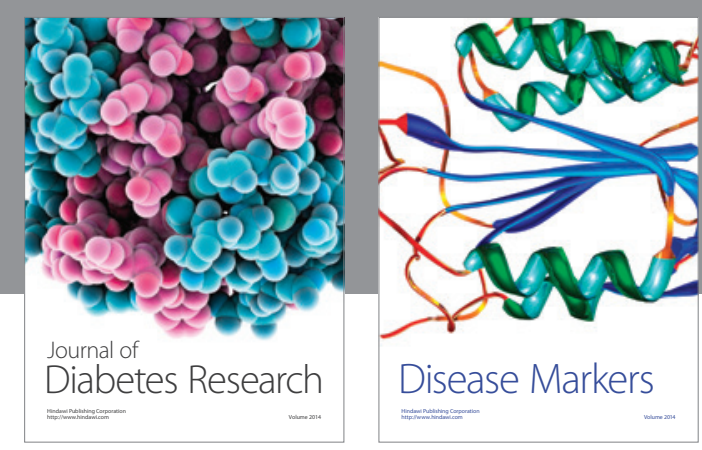

Disease Markers
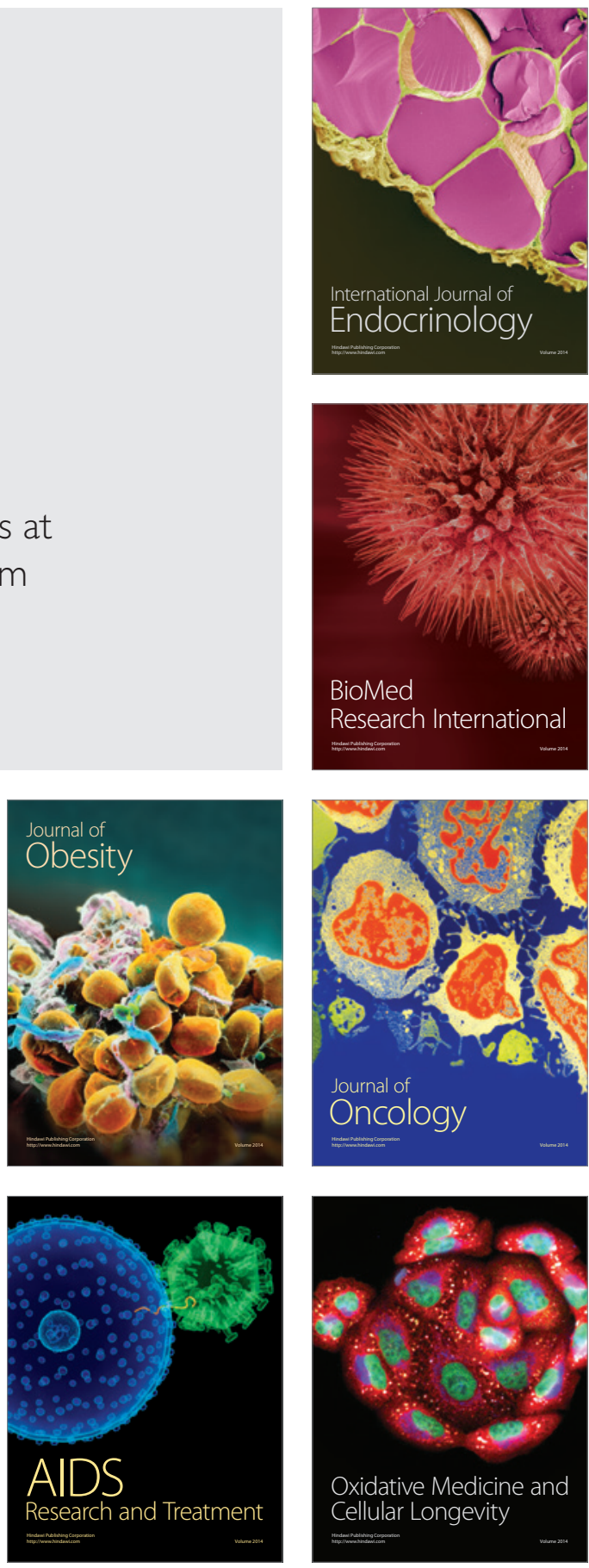